Obere Extremität 2012 $\cdot 7: 188$

DOI 10.1007/s11678-012-0194-9

Online publiziert: 9. November 2012

(c) Springer-Verlag Berlin Heidelberg 2012

\section{Joachim Windolf}

Klinik für Unfall- und Handchirurgie, Universitätsklinikum Düsseldorf, Düsseldorf, Deutschland

\section{Distale Radiusfrakturen}

Rund 200 Jahre nach der Beschreibung des körperfernen Speichenbruchs an typischer Stelle durch Abraham Colles 1814 gibt es eine Vielzahl unterschiedlicher Verfahren zur Behandlung der distalen Radiusfraktur. Meilensteine in der Entwicklung der geschlossenen Frakturbehandlung waren die Reposition und Ruhigstellung nach Böhler, die Bohrdrahtosteosynthese nach Willenegger und die intrafokale K-Draht-Osteosynthese in der Technik nach Kapandji. Besondere Bedeutung erlangte auch der Fixateur externe, der unter Nutzung der Ligamentotaxis eine stabile Retention erzielt und insbesondere zur Stabilisierung von Trümmerfrakturen oder offenen Verletzungen und in der Versorgung polytraumatisierter Patienten seinen festen Stellenwert hat.

Die offene Frakturbehandlung entwickelte sich gegenüber den geschlossenen Verfahren nur zögerlich. Sie blieb zunächst auf Schraubenosteosynthesen partieller Gelenkverletzungen und abstützende Plattenosteosynthesen von Flexionsfrakturen begrenzt. Plattenosteosynthesen von Extensionsfrakturen mit dorsaler Trümmerzone konnten sich in Ermangelung entsprechend dimensionierter Implantate aufgrund der Zugangsmorbidität und der häufigen Repositionsverluste lange Zeit nicht durchsetzen. Günstiger erschien hier die Fixation der distalen Fragmente mit Kirschner-Drähten, Auffüllung der Defekte mit Spongiosa und Stabilisierung der Frakturzone mit einem Fixateur externe.

Seit Einführung der winkelstabilen Plattenosteosynthese von palmar hat sich die Versorgungsrealität distaler Radiusfrakturen grundlegend gewandelt. Sie führt auch bei instabilen Frakturtypen ohne Defektauffüllung zu guten funktionellen und radiologischen Ergebnissen. Die in der Literatur anfänglich beschrie- bene hohe Komplikationsrate ließ sich nach sorgfältiger Analyse der berichteten Probleme mit einer differenzierten Indikationsstellung, exakter Operationstechnik und weiter verbesserten Implantaten deutlich senken. Insbesondere Verletzungen der Sehnen lassen sich durch eine exakte Platzierung der Platte und korrekte Schraubenlängen vermeiden.

Aufgrund der meist deutlich verkürzten Behandlungsdauer mit frühzeitiger Wiederherstellung der Handgelenkfunktion wird die palmare winkelstabile Plattenosteosynthese vielerorts als Goldstandard zur Behandlung der distalen Radiusfraktur angesehen. Bislang gibt es aber keine validen Daten, die eine tatsächliche Überlegenheit des Verfahrens gegenüber anderen etablierten Verfahren belegen könnten. Im vorliegenden Themenschwerpunkt soll daher zunächst eine kritische Bilanz zur palmaren Plattenosteosynthese gezogen werden. Ein weiterer Beitrag gibt einen Überblick über neuere, minimal-invasive Verfahren, die eine besonders weichteilschonende und stabile Versorgung distaler Radiusfrakturen ermöglichen sollen. Die vorgestellten experimentellen Daten sollen helfen, das Potenzial solcher Verfahren als mögliche Konkurrenz zur winkelstabilen Platte einzuschätzen. Ein dritter Beitrag beschäftigt sich mit der Analyse etwaiger Bewegungseinschränkungen nach stattgehabter Radiusfraktur, die der konventionellen Bewegungsmessung durch manuelle Goniometrie verborgen bleiben können.

Obwohl die meisten distalen Radiusfrakturen und insbesondere die stabilen Frakturtypen eine günstige Prognose haben, kann es auch heute noch sowohl nach operativer als auch nach konservativer Therapie zu einem unbefriedigenden Behandlungsergebnis kommen. Wir bleiben daher aufgefordert, unsere etablierten Behandlungsstrategien kritisch zu hinter- fragen und neuere Entwicklungen aufmerksam zu prüfen.

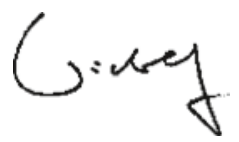

\section{Joachim Windolf}

\section{Korrespondenzadresse}

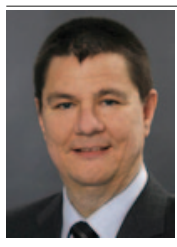

Prof. Dr. J. Windolf

Klinik für Unfall- und Handchirurgie Universitätsklinikum Düsseldorf Moorenstraße 5 40225 Düsseldorf windolf@uni-duesseldorf.de 